\title{
Cue-Induced Cocaine Seeking and Relapse Are Reduced by Disruption of Drug Memory Reconsolidation
}

\author{
Jonathan L. C. Lee, Amy L. Milton, and Barry J. Everitt \\ Department of Experimental Psychology, University of Cambridge, Cambridge CB2 3EB, United Kingdom
}

\begin{abstract}
Long-lasting vulnerability to drug cue-induced relapse to a drug-taking habit is a major challenge to the treatment of drug addiction. Here we show that blockade of drug memory reconsolidation, through infusion of Zif268 antisense oligodeoxynucleotides into the basolateral amygdala shortly before reexposure to a cocaine-associated stimulus but not simply to the training context, severely impaired subsequently cue-maintained cocaine seeking under a second-order schedule of reinforcement and abolished cue-induced reinstatement of and relapse to cocaine seeking. This reduction in relapse after disrupted memory reconsolidation was not only seen after several hundred pairings of the stimulus with self-administered cocaine, but older, as well as recent, memories were also disrupted. Reconsolidation blockade may thus provide a potential therapeutic strategy for the prevention of relapse in drug addiction.
\end{abstract}

Key words: addiction; relapse; memory reconsolidation; basolateral amygdala; antisense oligodeoxynucleotides; rat

\section{Introduction}

Drug addiction is often described as a chronic relapsing disorder, in which craving and relapse to drug seeking occur even after prolonged abstinence (Gawin and Kleber, 1986). A major contributor to relapse is exposure to environmental stimuli that have previously been associated regularly with the effects of selfadministered drugs. Such drug-associated stimuli evoke memories of the effects of the drug, induce craving in abstinent addicts, and precipitate relapse to a drug-taking habit (Gawin and Kleber, 1986; O’Brien et al., 1998; Childress et al., 1999). In experimental animals, a conditioned stimulus (CS) paired repeatedly with the self-administration of cocaine similarly induces relapse to, or reinstatement of, drug seeking (de Wit and Stewart, 1981; Meil and See, 1996; Fuchs et al., 1998; Grimm et al., 2001), while also supporting prolonged periods of cocaine seeking, for example under second-order schedules of reinforcement (Arroyo et al., 1998; Everitt and Robbins, 2000). Through association with the effects of cocaine, the CS acquires powerful conditioned reinforcing and other properties that are not easily diminished by nonreinforced presentations of the CS (Di Ciano and Everitt, 2004), and this may explain why cue exposure therapy in a clinical setting has not generally been a successful treatment strategy for drug addiction (Conklin and Tiffany, 2002).

Memories elicited by CSs are thought to reconsolidate after each episode of retrieval or reactivation, thereby allowing them to be updated, and disruption of this reconsolidation process results in a reduction in the motivational properties of stimuli associated

Received Sept. 29, 2005; revised April 21, 2006; accepted April 22, 2006

This work was supported by a grant from the Medical Research Council (United Kingdom) and was conducted within the Medical Research Council/Wellcome Trust Behavioural and Clinical Neuroscience Institute.

Correspondence should be addressed to Jonathan L. C. Lee, Department of Experimental Psychology, University of Cambridge, Downing Street, Cambridge CB2 3EB, UK. E-mail: jl12@cam.ac.uk.

D01:10.1523/JNEUROSCI.0323-06.2006

Copyright $\odot 2006$ Society for Neuroscience $\quad$ 0270-6474/06/265881-07\$15.00/0 previously with aversive outcomes (Nader et al., 2000). Reconsolidation requires de novo protein synthesis (Nader et al., 2000), with a critical role for the immediate-early gene Zif268 (also known as EGR1, NGFI-A, and Krox24) (Bozon et al., 2003; Lee et al., 2004). Infusion into the basolateral amygdala (BLA) of antisense oligodeoxynucleotides (ASO) targeting Zif268, which knocks down BLA Zif268 protein levels (Lee et al., 2005), disrupts the reconsolidation of discrete fear memories and greatly diminishes conditioned fear measured subsequently (Lee et al., 2005). We also demonstrated that CS-cocaine associations undergo reconsolidation in a process that is also critically dependent on Zif268 expression in the BLA, assessed in a procedure that isolates and measures the conditioned reinforcing properties of the cocaine cue (Lee et al., 2005).

Although it has been shown that the reconsolidation of drugrelated memories can be disrupted (Lee et al., 2005; Miller and Marshall, 2005), it remains to be demonstrated that impairment of drug memory reconsolidation, which is a potential treatment strategy for drug addiction, can in practice attenuate cueinduced maintenance of and relapse to cocaine seeking. Furthermore, it is important that the efficacy with which reconsolidation blockade can reduce relapse is tested after extensive drug self-administration as well as on old, or well established, drug memories when a substantial period of time has elapsed since the last episode of self-administration, because a prolonged previous drug-taking history is characteristic of abstinent addicts. Therefore, in the present experiments, we used second-order schedules of cocaine reinforcement (Di Ciano and Everitt, 2003) and a model of cue-induced cocaine craving (Lu et al., 2005) to investigate whether blocking the reconsolidation of CS-cocaine associations using intra-BLA infusions of Zif268 ASO before drug memory reactivation by CS reexposure in rats having self-administered cocaine would reduce subsequently cue-maintained cocaine seeking and cueinduced relapse. 


\section{Materials and Methods}

Subjects. The subjects were 100 experimentally naive adult male Lister Hooded rats, weighing 280-350 g. They were housed in pairs before surgery, and singly thereafter, in holding rooms maintained at $21^{\circ} \mathrm{C}$ on a reversed-light cycle (12 h light/dark cycle; lights on at 7:00 P.M.). After recovery from surgery, food was restricted to $20 \mathrm{~g} / \mathrm{d}$. Water was available ad libitum throughout the experiment. All procedures were conducted in accordance with the United Kingdom 1986 Animals (Scientific Procedures) Act (Project License PPL 80/1767).

Surgery and infusions. Rats were implanted with a single catheter in the right jugular vein and also with bilateral chronic indwelling guide cannulas targeting the BLA. The coordinates for cannula implantation were (relative to bregma) as follows (in $\mathrm{mm}$ ): anteroposterior, -2.6 ; mediolateral, \pm 4.5 ; dorsoventral, -5.6 (from dura). Details of the intravenous and stereotaxic surgical procedures have been described previously (Di Ciano and Everitt, 2004; Lee et al., 2004). A minimum of $7 \mathrm{~d}$ was allowed before behavioral training and testing began.

Infusions of Zif268 ASO/missense oligodeoxynucleotides (MSO) (1.0 $\mu \mathrm{l} / \mathrm{side} ; 0.125 \mu \mathrm{l} / \mathrm{min}$ ) were performed as described previously (Lee et al., 2005). Oligodeoxynucleotides were PAGE-purified phosphorothioate end-capped 18-mer sequences, resuspended in sterile PBS to a concentration of $2 \mathrm{nmol} / \mu \mathrm{l}$ as used previously (Lee et al., 2004, 2005): Zif268 ASO, 5' -GGT AGT TGT CCA TGG TGG-3'; Zif268 MSO, 5'-GTG TTC GGT AGG GTG TCA-3. We demonstrated previously using biotinylated oligodeoxynucleotides that these infusion parameters result in spread of Zif268 ASO/MSO restricted to the amygdala (Lee et al., 2005).

Cocaine self-administration. All behavioral procedures were performed in operant chambers (Med Associates, Lafayette, IN) as described previously (Di Ciano and Everitt, 2004) and were based on previous experiments (Di Ciano and Everitt, 2004; Lu et al., 2005). Rats underwent $10 \mathrm{~d}$ of cocaine self-administration training under a fixed-ratio 1 (FR1) schedule of reinforcement. At the start of each self-administration session, two levers were inserted into the operant chamber, and the house light was illuminated. Each response on the active lever (counterbalanced left or right) was reinforced with a single intravenous infusion of cocaine $(0.25$ $\mathrm{mg}$ in $0.1 \mathrm{ml}$ over $5 \mathrm{~s}$ per infusion), accompanied by a 20 s illumination of the CS light located above the active lever, during which both levers were retracted and the house light was extinguished. Responses on the other, inactive lever had no programmed consequence. "Priming" injections of cocaine were never given. To prevent accidental overdose, rats were limited to 30 infusions/h in the $3 \mathrm{~h}$ sessions.

Other groups of rats underwent $3 \mathrm{~d}$ of cocaine self-administration under an FR1 schedule, after which the interval schedule was increased daily through fixed-interval $1 \mathrm{~min}$ (FI1), FI2, FI4, FI8, and FI10, before stabilizing at FI15 for $3 \mathrm{~d}$. These groups had $2 \mathrm{~h}$ sessions with a limit of 30 infusions per session.

Drug memory reactivation. The memory for the CS-cocaine association was reactivated in a single $30 \mathrm{~min}$ session through 30 noncontingent presentations of the CS light (20 s; $40 \mathrm{~s}$ interstimulus interval). No levers were present during the reactivation session, which took place either 3 or $27 \mathrm{~d}$ after the completion of self-administration training and $90 \mathrm{~min}$ after infusion of Zif268 ASO or MSO into the BLA. Nonreactivated control context reexposed groups were infused with Zif $268 \mathrm{ASO}$ or MSO into the BLA 90 min before a 30 min session involving reexposure to the training context alone (no CS presentations). Such nonreactivated controls were only included for the $6 \mathrm{~d}$ condition of the relapse procedure and for the maintenance of cocaine seeking under a second-order schedule of reinforcement, and the consistent observation that infusion of Zif268 ASO into the BLA in the absence of memory reactivation has no effect on subsequent behavior obviated the use of this control for every behavioral condition. Similarly, delayed infusion control groups were only included for the $6 \mathrm{~d}$ condition in the relapse procedure, in which rats were infused $4 \mathrm{~h}$ after the end of the reactivation session, such that the infusions were in fact delayed by $6 \mathrm{~h}$.

Cue-induced cocaine seeking. Testing, which took place 6 or $30 \mathrm{~d}$ after self-administration, involved the levers again being extended into the operant chamber (Lu et al., 2005). A response on the active lever was reinforced by a $1 \mathrm{~s}$ presentation of the CS light, during which the house light was extinguished because brief presentations of a pavlovian CS are optimal for it to act as a conditioned reinforcer (Mackintosh, 1974), and a response on the inactive lever again had no programmed consequence. There were no cocaine infusions, and the number of lever presses were recorded in two $30 \mathrm{~min}$ bins of a $60 \mathrm{~min}$ session.

Rats infused with Zif268 ASO or MSO were allocated at random to two experimental conditions ( $6 \mathrm{~d}$ and $30 \mathrm{~d}$ ). Rats in the $6 \mathrm{~d}$ condition were infused and reactivated $3 \mathrm{~d}$ after training and $3 \mathrm{~d}$ before test (thus being tested $6 \mathrm{~d}$ after training). An additional group of rats (no CS) were infused with Zif268 MSO and reactivated, but the CS was omitted during the test. Rats in the $30 \mathrm{~d}$ condition were tested $30 \mathrm{~d}$ after training but infused and reactivated $3 \mathrm{~d}$ before test. Within each condition, the rats were also randomly assigned to Zif268 ASO or MSO treatment.

Maintenance of cocaine seeking under a second-order schedule of reinforcement. The groups of rats maintained under an FI15 schedule of cocaine reinforcement (baseline) were infused and reactivated as above and, on the next day, were tested under a second-order schedule of reinforcement [FI15(FR10:S)], in which a $1 \mathrm{~s}$ CS was introduced contingently on each 10th active lever press, and the first CS presentation (20 s) after the $15 \mathrm{~min}$ interval was paired with an infusion of cocaine (Di Ciano and Everitt, 2003). After eight sessions under FI15(FR10:S), the CS was omitted for five sessions (FI15) and then reintroduced for 3 sessions [FI15(FR10:S)].

Reinstatement of extinguished cocaine seeking. Finally, after the completion of the second-order schedule of reinforcement phase, an extinctionreinstatement procedure was initiated, in which the instrumental lever press response was extinguished in the absence of the CS to a criterion of $<50$ responses on the active lever in $2 \mathrm{~h}$. On the next day, rats were tested for cue-induced reinstatement of cocaine seeking (day 50 after memory reactivation). Each active lever response was reinforced by a $1 \mathrm{~s}$ presentation of the CS light in a 60 min session, in which no cocaine was available.

Histology. After completion of behavioral testing, the rats were perfused, and their brains were sectioned coronally at $60 \mu \mathrm{m}$. Sections were stained with cresyl violet. Cannula placements were then assessed using light microscopy by an observer blind to the behavioral results. Subjects were only included in the statistical analysis if the injectors were located bilaterally within the BLA, and there was no bilateral damage to the amygdala or any other area of the brain.

Statistical analysis. Data are presented as mean \pm SEM number of responses on the active and inactive levers. Three- or four-way repeatedmeasures ANOVAs were performed on the data with factors ASO, reactivation, condition, bin, lever (active vs inactive), session, and interval (before cocaine vs after cocaine for the maintenance experiment) as appropriate. Planned comparisons included the total number of CS-drug pairings during self-administration. To compare the effects of Zif268 ASO-induced impairments in responding and the omission of the CS during the relapse test, the $6 \mathrm{~d}$ MSO and ASO groups were analyzed with the no-CS group in a four-way repeated-measures ANOVA including reactivation as a factor. Furthermore, the groups infused with Zif268 MSO and then reexposed to the CS or just the context were compared to assess any extinction. Data from representative sessions from the maintenance experiment [baseline FI15, FI15(FR10:S) CS introduction, FI15 CS omission, and FI15(FR10:S) CS reintroduction] were analyzed in a four-way repeated-measures ANOVA, with planned comparisons including an analysis of the ASO infused group alone. Tukey's test was selected for post hoc analyses, and a significance level of $p<0.05$ was selected for all analyses.

\section{Results \\ Blocking reconsolidation abolishes cue-induced cocaine seeking}

All rats included in the behavioral analyses had cannulas placed bilaterally in the BLA (Fig. 1). There was no difference between any of the groups during cocaine self-administration training, and, on average, rats received 505 pairings of the CS with an infusion of cocaine (data not shown; condition $\times$ ASO, $F<1$ ).

A single infusion of Zif268 ASO into the BLA, $3 \mathrm{~d}$ after the last 


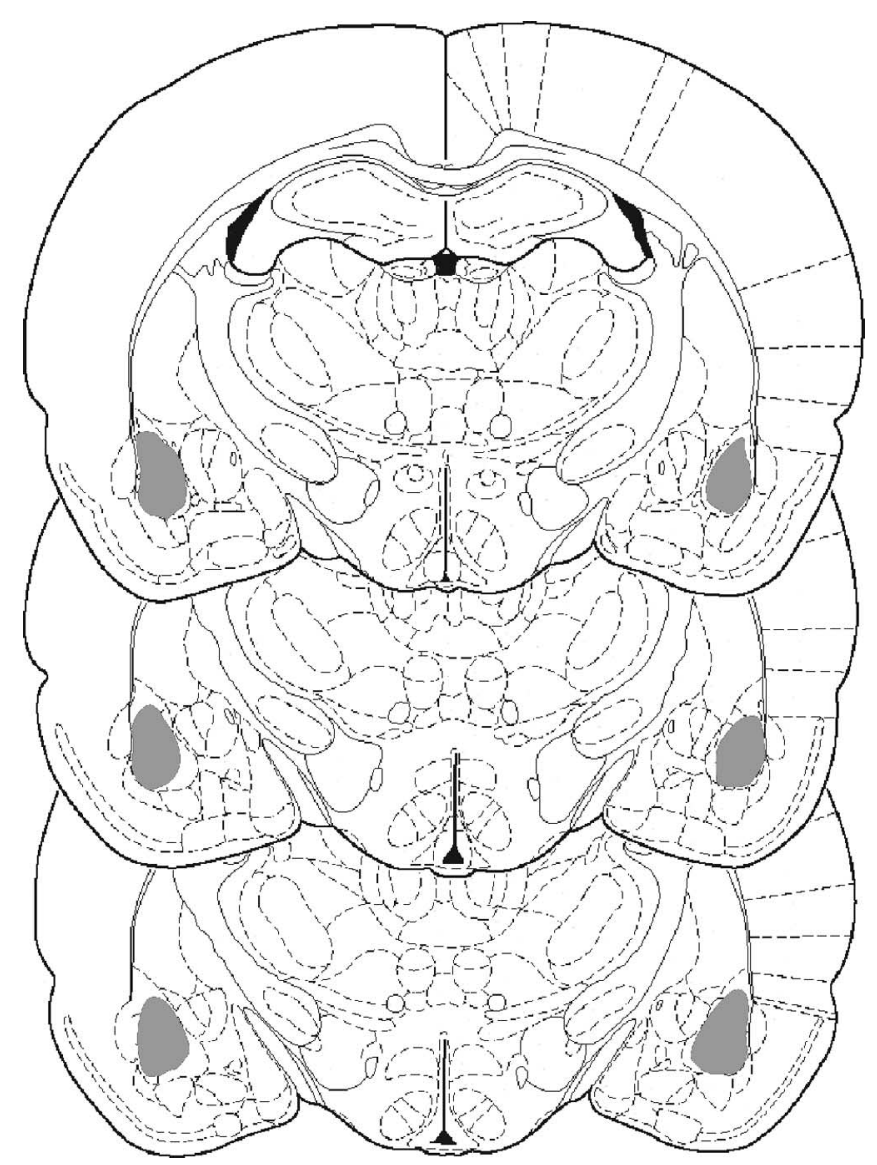

Figure 1. Location of injectors within the BLA. Schematic representation of the brain at three rostrocaudal levels $(-2.30,-2.56$, and $-2.80 \mathrm{~mm}$ from bregma). All rats included in the statistical analyses had injectors placed bilaterally in the BLA (gray shaded area).

cocaine self-administration, abolished subsequent cue-induced drug seeking 3 d later, i.e., on day 6 after the end of training. This effect was critically dependent on reactivation of the CS-cocaine memory by reexposure to the cocaine-associated CS and not just to the training context (Fig. $2 a, b$ ). The reexposure to the CS itself did not induce any evidence of extinction because there was no reactivation $\times$ lever interaction for the groups infused with Zif268 MSO $(F<1)$. Infusion of Zif268 ASO, but not control Zif268 MSO, significantly impaired discriminated responding throughout the $1 \mathrm{~h}$ session. An ANOVA revealed a significant reactivation $\times$ condition (ASO vs $\mathrm{MSO}$ vs no $\mathrm{CS}) \times$ lever interaction $\left(F_{(2,40)}=4.75 ; p<0.02\right)$ with no reactivation $\times$ condition $\times$ bin $\times$ lever interaction $(F<1)$, and analysis of the context reexposed groups alone showed that the no-CS group was impaired relative to the Zif268 ASO and MSO groups, which did not differ from each other (condition $\times$ lever, $F_{(2,18)}=4.44, p<0.03$; condition $\times$ bin $\times$ lever, $F<1$, post hoc $p<0.05$ ). Additional analysis revealed that Zif268 ASO infusion impaired discriminated responding reactivation dependently relative to Zif268 MSO infusion (reactivation $\times$ condition $\times$ lever, $F_{(1,31)}=6.59$, $p<0.02$; reactivation $\times$ condition $\times$ bin $\times$ lever, $F_{(1,31)}=1.18$, $p>0.28$ ). In contrast, the no-CS group was impaired relative to infusion of Zif268 MSO regardless of reactivation (condition $X$ lever, $F_{(1,24)}=13.05, p<0.01$; reactivation $\times$ condition $\times$ lever, $F<1$; reactivation $\times$ condition $\times$ bin $\times$ lever, $F<1$ ) and differed from the Zif268 ASO infused group in a reactivationdependent manner (reactivation $\times$ condition $\times$ lever, $F_{(1,25)}=$ 7.86, $p<0.02$; reactivation $\times$ condition $\times$ bin $\times$ lever, $F_{(1,25)}=$
$2.00, p>0.16)$. Importantly, analysis of the CS reexposed groups showed that the Zif268 ASO and no-CS groups did not differ from each other, but both were impaired relative to the Zif268 MSO group (condition $\times$ lever, $F_{(2,22)}=11.15, p<0.01$; condition $\times$ bin $\times$ lever, $F<1$, post hoc $p<0.05$ ). Therefore, the reduction in drug seeking after amygdala Zif268 knockdown at the time of memory reactivation was both qualitatively and quantitatively identical to omitting the CS completely (Fig. 2c). Prereactivation infusion of Zif268 ASO $3 \mathrm{~d}$ after training in fact persistently reduced subsequent cocaine seeking for at least $41 \mathrm{~d}$ (supplemental Fig. 1, available at www.jneurosci.org as supplemental material).

The effect of Zif268 ASO was not observed when the infusion was delayed by $6 \mathrm{~h}$, thus taking place $4 \mathrm{~h}$ after the end of the CS reexposure session (Fig. 2d). ANOVA revealed no ASO $\times$ lever or ASO $\times$ bin $\times$ lever interactions or a main effect of ASO $(F$ values $<1)$. Therefore, there is a limited time window of opportunity during which the reconsolidation of the reactivated drug memory can be disrupted by infusion of Zif268 ASO into the BLA.

Zif268 ASO also reduced drug seeking when the drug memory was reactivated $27 \mathrm{~d}$ after the last session of cocaine selfadministration, the rats being tested after more protracted cocaine withdrawal (Fig. 3). Discriminated responding was significantly reduced in Zif268 ASO infused rats compared with Zif268 MSO infused controls. An ANOVA revealed a significant ASO $\times$ lever interaction $\left(F_{(1,12)}=5.93 ; p<0.04\right)$, with no ASO $\times$ bin $\times$ lever interaction $(F<1)$, demonstrating that the Zif268 ASOinduced impairment was observed throughout the $1 \mathrm{~h}$ test session. Therefore, old as well as more recent drug memories were disrupted by impairing memory reconsolidation.

\section{"Incubation" of cocaine cue-reinforced responding and cocaine seeking}

An overall ANOVA (including both 6 and $30 \mathrm{~d}$ conditions) revealed both a significant condition $\times$ lever interaction $\left(F_{(1,30)}=23.65 ; p<0.01\right)$ and a significant main effect of condition $\left(F_{(1,30)}=11.29 ; p<0.01\right)$. This analysis revealed a timedependent elevation of responding in withdrawal from cocaine, which adds additional support to the evidence showing the incubation of responding in both the presence (Lu et al., 2005) and absence (Grimm et al., 2001) of the CS. Moreover, there were no condition $\times$ lever $\times$ ASO $(F<1)$ or condition $\times$ lever $\times$ ASO $\times$ bin $\left(F_{(1,30)}=1.45 ; p>0.23\right)$ interactions, indicating that the disruptive effect of Zif268 ASO did not differ when the drug memory was old or more recent.

\section{Blocking reconsolidation abolishes the ability of a cocaine- associated CS to enhance cocaine seeking under a second- order schedule of reinforcement}

There was no difference between any of the groups during cocaine self-administration training, and, on average, rats received 210 pairings of the CS with an infusion of cocaine before drug memory reactivation (data not shown; condition $\times$ ASO, $F<1$ ).

Infusion of Zif268 ASO before drug memory reactivation prevented the CS from subsequently increasing and maintaining cocaine seeking at an enhanced level under a second-order schedule of reinforcement, an effect that was again dependent on reexposure to the cocaine-associated CS (Fig. 4). ANOVA revealed a significant reactivation $\times$ ASO $\times$ lever $\times$ session interaction $\left(F_{(3,78)}=4.75 ; p<0.01\right)$, with no reactivation $\times$ ASO $\times$ lever $\times$ session $\times$ interval interaction $(F<1)$, indicating that Zif268 ASO impaired discriminated responding in a reactivation-dependent manner. Importantly, there were no differences between the 
groups during both baseline and CS omission because, during these sessions, there were no reactivation $\times$ ASO $\times$ lever or reactivation $\times$ ASO $\times$ lever $\times$ bin interactions $(F$ values $<1)$. Furthermore, responding during CS omission was not different from baseline $(F$ values $<1)$.

Subsequent analysis demonstrated that Zif268 ASO during memory reactivation subsequently prevented the elevation of responding after both CS introduction under an FI15(FR10:S) second-order schedule of reinforcement relative to baseline (reactivation $\times$ ASO $\times$ lever $\times$ session, $F_{(1,26)}=5.78, p<0.01$; reactivation $\times$ ASO $\times$ lever $\times$ session $\times$ interval, $F<1$ ) and CS reintroduction relative to CS omission (reactivation $\times$ ASO $\times$ lever $\times$ session, $F_{(1,26)}=8.49, p<0.01$; reactivation $\times$ ASO $\times$ lever $\times$ session $\times$ interval, $\left.F_{(1,26)}=1.02, p>0.32\right)$. Thus, whereas CS introduction elevated responding by $138 \%$ in control and nonreactivated rats (and CS omission resulted in a reduction back to baseline levels of responding), reactivated Zif268 ASO infused rats were completely insensitive to the introduction and omission of the CS across several test sessions (lever $\times$ session, $F<1$; lever $\times$ session $X$ interval, $F<1$ ). Thus, the reduction in cocaine seeking after Zif268 knockdown in the BLA was qualitatively and quantitatively identical to omission of the CS completely. Therefore, the impact of cocaine cues to enhance cocaine seeking under a second-order schedule of reinforcement was abolished by previous disruption of the reconsolidation of the CS-cocaine association.

All of the analyses presented include interval as a factor, comparing responding during the first pre-cocaine interval with an average of the four post-cocaine intervals (i.e., periods after cocaine had been self-administered), the significant main effects of which $(p$ values $<0.01)$ demonstrate that cocaine consistently potentiated responding, confirming our previous observations (Arroyo et al., 1998; Di Ciano and Everitt, 2003). However, there were no reactivation $\times$ ASO $\times$ lever $\times$ session $\times$ interval interactions (see above), indicating that the cocaine potentiation of responding did not differ between groups and across sessions. Thus, Zif268 ASO-mediated blockade of drug memory reconsolidation reduced subsequent cocaine seeking under a secondorder schedule of reinforcement in both the pre-cocaine interval and the cocaine-potentiated intervals.

\section{Blocking reconsolidation abolishes cue-induced reinstatement of cocaine seeking}

After extinction of the instrumental response, cue-induced reinstatement of cocaine seeking was also abolished by the same previous blockade of drug memory reconsolidation (Fig. 5). Comparison of responding during reinstatement relative to the last session of extinction revealed a significant ASO $\times$ lever $\times$ session interaction $\left(F_{(1,15)}=4.67 ; p<0.05\right)$, with no ASO $\times$ lever interaction during extinction $(F<1)$. Analysis of the Zif268 ASO infused rats alone revealed no lever $\times$ session interaction $\left(F_{(1,7)}=\right.$ $1.28 ; p>0.29)$, indicating that these rats were completely insen-
C

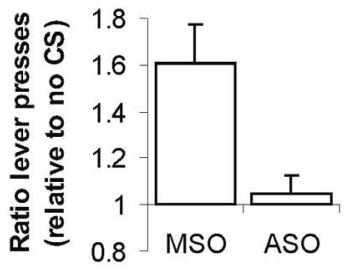

d

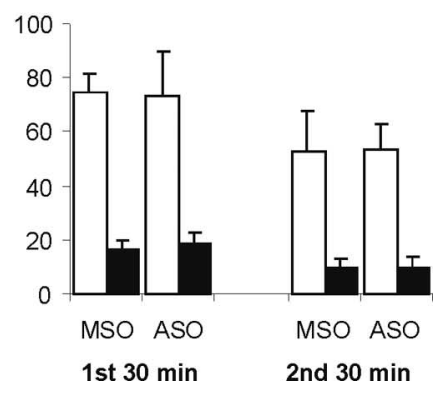

Figure 2. Effect of pre-reactivation intra-BLA Zif268 ASO on subsequent cocaine seeking 6 d into withdrawal. $\boldsymbol{a}$, The number of infusions of Zif268 ASO and MSO after reexposure to the cocaine CS ( $n=4$ per group). Data are presented as mean + SEM.

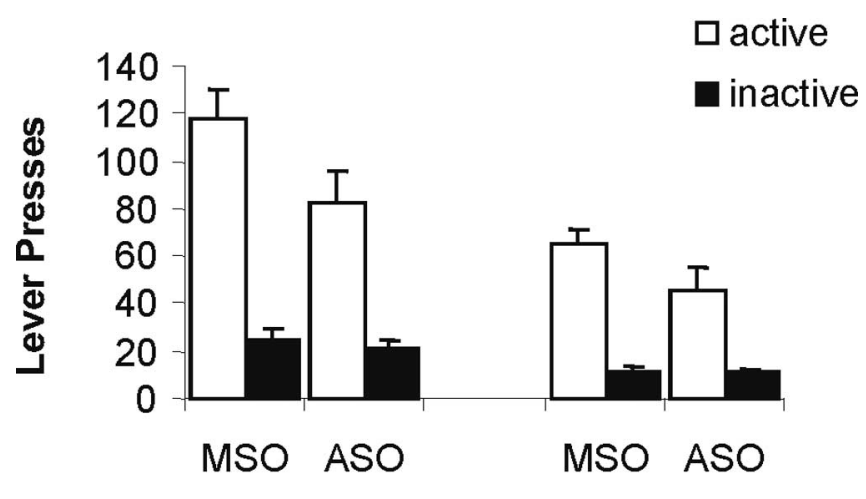

$1 \mathrm{st} 30 \mathrm{~min}$

2nd $30 \mathrm{~min}$

Figure 3. Effect of pre-reactivation intra-BLA Zif268 ASO on subsequent cocaine seeking 30 dinto withdrawal. The number of active and inactive lever presses during the first and second 30 min bins of the 60 min test are presented for rats infused $27 \mathrm{~d}$ after the end of selfadministration training ( $n=6$ and 8 per group). Data presented as mean + SEM.

sitive to the contingent introduction of the CS during the reinstatement test.

\section{Discussion}

The present results demonstrate that Zif268 ASO-induced blockade of drug memory reconsolidation in the amygdala persistently impairs the maintenance of cocaine seeking as well as relapse to a previously established drug-seeking behavior, with no evidence of spontaneous recovery. Indeed, for recent drug memories, intra-BLA Zif268 ASO infusion at CS-cocaine memory reactivation subsequently reduced responding to the same extent as completely omitting the CS during test, indicating that it effectively abolished the conditioned reinforcing properties of the CS and thereby its ability to support drug-seeking responses. Further- 

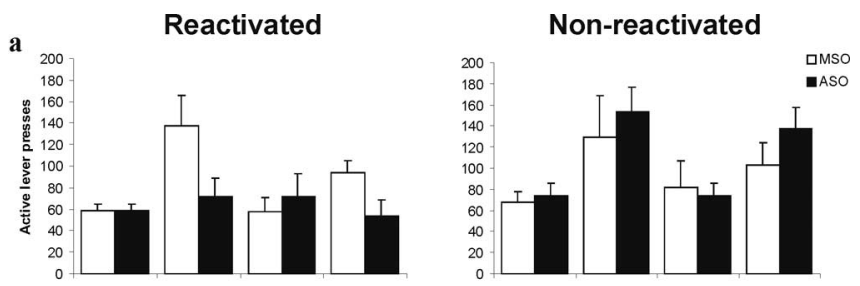

b
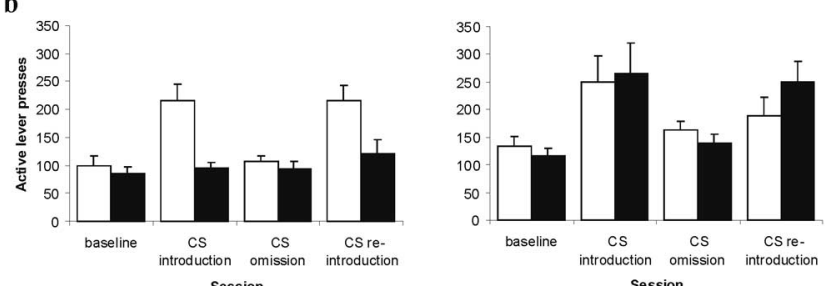

Figure 4. Effect of pre-reactivation intra-BLA Zif268 ASO on the subsequent maintenance of cocaine seeking under a second-order schedule of reinforcement. The number of active lever presses during the first, pre-cocaine interval ( $\boldsymbol{a}$ ) and averaged over the four post-cocaine intervals $(\boldsymbol{b})$ are presented for representative sessions in the four stages of the experiment $(n=$ 6-10 per group): baseline, CS introduction under a second-order schedule of reinforcement [FI15(FR10:S); session 6 after reactivation], CS omission (FI15; session 13), and CS reintroduction (session 15). Groups were infused with Zif268 ASO or MSO and reexposed to either the training context alone (Non-reactivated) or the cocaine-associated CS in the training context (Reactivated). Infusion and reexposure took place between baseline and CS introduction. Data are presented as mean \pm SEM.

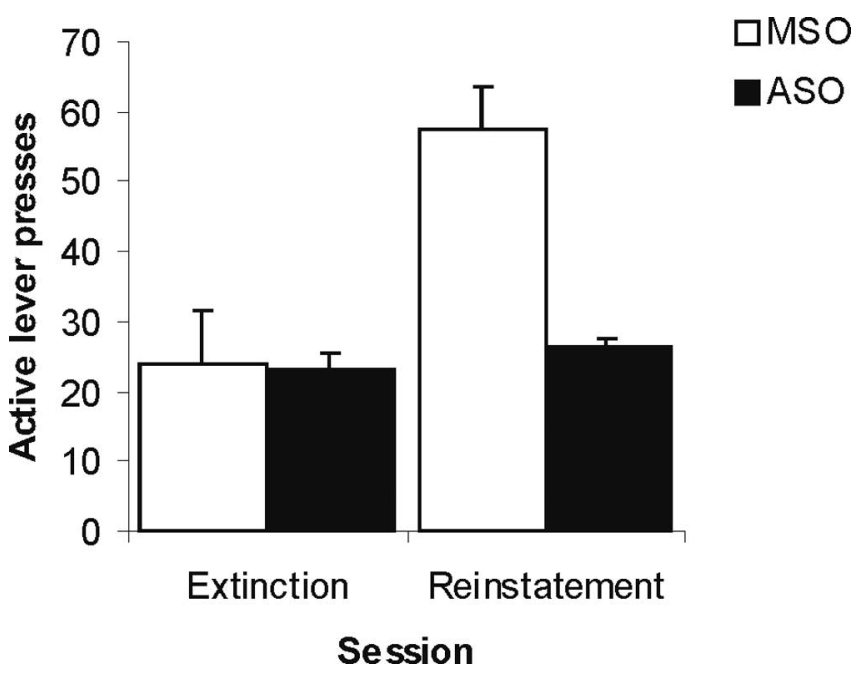

Figure 5. Effect of pre-reactivation intra-BLA Zif268 ASO on the subsequent cue-induced reinstatement of cocaine seeking after extinction of the instrumental response. The number of active and inactive lever presses are presented for the first hour of the last session of instrumental extinction and for the subsequent $1 \mathrm{~h}$ reinstatement test ( $n=8-9$ per group). Data are presented as mean + SEM.

more, reconsolidation blockade also reduced relapse when the age of the drug memory was increased to $27 \mathrm{~d}$. Thus, even longestablished drug memories are vulnerable to disruption when reactivated during a single session of cocaine-associated CS presentation, such that the vigor or reinstatement of drug seeking is greatly reduced.

Through repeated pairings with the effects of cocaine, a previously neutral environmental stimulus acquires enduring conditioned reinforcing properties that maintain prolonged periods of drug seeking, induce craving, and precipitate relapse (Meil and See, 1996; Arroyo et al., 1998; Lu et al., 2005). The acquisition of conditioned reinforcing properties depends on pairings of the CS with natural reward or drug, because it is well established that explicitly unpaired CSs are ineffective conditioned reinforcers (Taylor and Robbins, 1984; Di Ciano and Everitt, 2003, 2004; Parkinson et al., 2005). A specific test of the conditioned reinforcing properties of a drug-associated CS is its ability to support the acquisition of a new response (Mackintosh, 1974; Di Ciano and Everitt, 2004). In a previous study, we showed that intra-BLA Zif268 ASO infusion before reexposure to a cocaine-paired CS abolished the subsequent ability of the CS to support this new learning (Lee et al., 2005). Therefore, Zif268 ASO disrupted the reconsolidation of the original CS-cocaine association, thereby preventing the CS from subsequently acting as a conditioned reinforcer (Lee et al., 2005). Here we greatly extended this initial observation to show the major impact of drug memory reconsolidation blockade using well established and translationally valid models of drug seeking, craving, and relapse (Arroyo et al., 1998; Grimm et al., 2001; Di Ciano and Everitt, 2003; Lu et al., 2005). We demonstrate that blocking drug memory reconsolidation abolishes subsequently both the ability of a drug-associated CS to maintain and reinstate cocaine seeking and the major component of relapse to a previously established drug-seeking behavior that is precipitated by exposure to a cocaine-associated CS.

The BLA is a primary locus of CS-drug associations (Everitt et al., 2000), and excitotoxic lesions of the BLA both impair the ability of cocaine-associated stimuli to maintain responding under a second-order schedule of reinforcement (Whitelaw et al., 1996) and reduce cocaine cue-induced reinstatement of drug seeking (Meil and See, 1997). The BLA is important for conditioned reinforcement and outcome-specific incentive processes (Burns et al., 1993; Hatfield et al., 1996; Everitt et al., 2000; Corbit and Balleine, 2005), whereas the central nucleus of the amygdala $(\mathrm{CeN})$ is not (Robledo et al., 1996; Everitt et al., 2000; Corbit and Balleine, 2005). Furthermore, reexposure to a cocaine-associated stimulus specifically upregulates the expression of Zif268 in the BLA but not the CeN (Thomas et al., 2003). Local infusion of Zif268 ASO knocks down Zif268 protein levels in the BLA (Lee et al., 2005). Therefore, the present impairment in cue-induced maintenance of and relapse to cocaine seeking most likely results from the knockdown of Zif268 protein levels in the BLA.

Our previous study showed that the effect of intra-amygdala Zif268 ASO to reduce cocaine seeking was critically dependent on the reactivation session, in which rats were reexposed to the CS in the training context (Lee et al., 2005). Here, in the recent drug memory condition for the relapse procedure and under a secondorder schedule of reinforcement, we not only confirmed the reactivation dependence of the effects of Zif268 ASO but further demonstrated that it is reexposure to the CS itself, rather than simply to the training context, that is necessary to reactivate the CS-drug memory and render it vulnerable to disruption. IntraBLA infusion of Zif268 ASO before reexposure to the context alone had no effect on subsequent cocaine seeking. Therefore, reexposure to the context alone does not reactivate the CS- Cocaine memory, consistent with a recent finding that only directly reactivated memories undergo reconsolidation in the amygdala (Debiec et al., 2006). Furthermore, we used the relapse procedure and 3-d-old drug memories to demonstrate that the disruptive effect of Zif268 ASO was abolished by delaying the infusion by $6 \mathrm{~h}$, so that it took place $4 \mathrm{~h}$ after the end of the reactivation session. Thus, there is a limited time window during which the reconsolidation of drug memories is vulnerable to disruption, which is consistent with studies of fear memory reconsolidation (Nader et al., 2000).

In contrast to previous studies (Lee et al., 2005; Miller and 
Marshall, 2005), the reactivation session used here involved rats that were exposed noncontingently to the CS. The observation that drug memory reconsolidation can be blocked after passive (i.e., pavlovian) exposure to the drug-associated stimuli that had previously been presented response-contingently during cocaine self-administration is especially important for any potential use of reconsolidation blockade in a therapeutic setting. Indeed, noncontingent presentation of cocaine-related stimuli has been shown to activate the amygdala and induce craving in humans (Grant et al., 1996; Childress et al., 1999; Garavan et al., 2000).

Zif268 ASO-infused rats were insensitive to the presence or absence of the CS during many days of behavioral testing. Under a second-order schedule of reinforcement, the impairment in cocaine seeking was persistent, lasting for at least $50 \mathrm{~d}$ (and at least $41 \mathrm{~d}$ in the relapse procedure), thereby extending our previous findings using an acquisition of a new response procedure (Lee et al., 2005). Furthermore, during the maintenance of cocaine seeking, there was no evidence of spontaneous recovery or cocaineinduced reinstatement of the CS-cocaine association. Although these results are consistent with those from the relapse procedure, the lack of reconditioning during the maintenance of cocaineseeking tests is perhaps somewhat surprising. The rats received on average 65 CS-cocaine pairings during the CS introduction and omission stages of the second-order schedule of reinforcement after the memory reactivation session, yet subsequent CS reintroduction had no effect on responding in the group in which the reconsolidation of the CS-cocaine association had been disrupted previously under either a second-order schedule of reinforcement or continuous reinforcement in the reinstatement test. It is possible that the low density of cocaine reinforcement under the fixed-interval $15 \mathrm{~min}$ of the second-order schedule may result in even this number of pairings being ineffective in conditioning a CS-cocaine association capable of supporting instrumental behavior subsequently when the CS is presented responsecontingently as a conditioned reinforcer. Alternatively, it may be the case that disruption of the reconsolidation of a previously conditioned CS-cocaine association also results in a deficit or delay in reconditioning. Additional studies will be required to investigate the potential effects of reconsolidation blockade on subsequent new learning.

Not unexpectedly, both the Zif268 ASO-infused and CSomitted groups displayed residual, but impaired, responding in the first $30 \mathrm{~min}$ of the relapse test, which then extinguished in the second half of the session. Although clearly not influenced by CS-cocaine associations because the effects of Zif268 ASO infusion were qualitatively and quantitatively identical to omission of the CS, this residual responding in extinction reflects previous instrumental learning. Therefore, blockade of CS-cocaine memory reconsolidation alone, although completely eliminating the impact of CSs on relapse, may not be able fully to abolish drug seeking. However, this manipulation does diminish the ability of drug cues to initiate relapse and instrumental drug-seeking responses. Clearly, methods to reduce the impact of instrumental learning may also contribute to the effectiveness of relapse prevention. Indeed, when rats had previously undergone extinction of the instrumental drug-seeking response, reconsolidation blockade resulted in a complete loss of effect of contingent cocaine cue introduction on the reinstatement of responding.

Many variables can impact on the induction and disruption of reconsolidation, including an interaction between the strength and age of the memory and the extent of stimulus reexposure (Eisenberg et al., 2003; Suzuki et al., 2004). Stronger and older aversive memories are more difficult to disrupt (Suzuki et al.,
2004). The very nature of drug addiction, which develops through the chronic self-administration of drugs, indicates that drug memories will inevitably be both well established (old) and strong. Therefore, the conditioning of the CS-cocaine association in the present relapse study was designed to occur through multiple sessions of cocaine self-administration that resulted in a number of pairings far greater than in previous studies (Lee et al., 2005; Miller and Marshall, 2005; Valjent et al., 2006). However, even under such conditions, limited stimulus reexposure in a single session was sufficient to reactivate the drug memory so that its subsequent reconsolidation could be disrupted by intraamygdala Zif268 ASO. Furthermore, in contrast to studies in which 14-d-old inhibitory avoidance memories in rats (Milekic and Alberini, 2002) and fear memories in medaka fish (Eisenberg and Dudai, 2004) were resistant to reconsolidation impairment, 45-d-old contextual fear memories were susceptible to reconsolidation impairment (Debiec et al., 2002). The present results additionally demonstrate that the reconsolidation of a well learned drug memory can be impaired not only at 3 but at $27 \mathrm{~d}$ after its acquisition. Together, these results indicate that neither the extent of drug experience nor the time that has elapsed since the onset of self-administration, both of which are characteristically substantial in addicts, are major obstacles to considering the use of reconsolidation blockade as a potential treatment strategy for drug addiction. However, the quantitative and temporal limits of these variables warrant additional detailed study.

In summary, rats were trained to self-administer cocaine over several days and received hundreds of CS-cocaine pairings. Nevertheless, through a single brief session of passive reexposure to the cocaine-associated stimulus, preceded by an infusion of Zif268 ASO into the BLA, the reconsolidation of both young and old drug memories was severely impaired to reduce cue-induced cocaine seeking and relapse. Thus, disruption of drug memory reconsolidation may be a powerful treatment strategy for the reduction of relapse in abstinent drug addicts.

\section{References}

Arroyo M, Markou A, Robbins TW, Everitt BJ (1998) Acquisition, maintenance and reinstatement of intravenous cocaine self-administration under a second-order schedule of reinforcement in rats: effects of conditioned cues and continuous access to cocaine. Psychopharmacology 140:331-344

Bozon B, Davis S, Laroche S (2003) A requirement for the immediate early gene zif268 in reconsolidation of recognition memory after retrieval. Neuron 40:695-701.

Burns LH, Robbins TW, Everitt BJ (1993) Differential-effects of excitotoxic lesions of the basolateral amygdala, ventral subiculum and medial prefrontal cortex on responding with conditioned reinforcement and locomotor-activity potentiated by intraaccumbens infusions of D-amphetamine. Behav Neurosci 55:167-183.

Childress AR, Mozley PD, McElgin W, Fitzgerald J, Reivich M, O’Brien CP (1999) Limbic activation during cue-induced cocaine craving. Am J Psychiatry 156:11-18.

Conklin CA, Tiffany ST (2002) Applying extinction research and theory to cue-exposure addiction treatments. Addiction 97:155-167.

Corbit LH, Balleine BW (2005) Double dissociation of basolateral and central amygdala lesions on the general and outcome-specific forms of pavlovian-instrumental transfer. J Neurosci 25:962-970.

de Wit H, Stewart J (1981) Reinstatement of cocaine-reinforced responding in the rat. Psychopharmacology 75:134-143.

Debiec J, LeDoux JE, Nader K (2002) Cellular and systems reconsolidation in the hippocampus. Neuron 36:527-538.

Debiec J, Doyere V, Nader K, LeDoux JE (2006) Directly reactivated, but not indirectly reactivated, memories undergo reconsolidation in the amygdala. Proc Natl Acad Sci USA 103:3428-3433.

Di Ciano P, Everitt BJ (2003) Differential control over drug-seeking behav- 
ior by drug-associated conditioned reinforcers and discriminative stimuli predictive of drug availability. Behav Neurosci 117:952-960.

Di Ciano P, Everitt BJ (2004) Conditioned reinforcing properties of stimuli paired with self-administered cocaine, heroin or sucrose: implications for the persistence of addictive behaviour. Neuropharmacology 47:202-213.

Eisenberg M, Dudai Y (2004) Reconsolidation of fresh, remote, and extinguished fear memory in medaka: old fears don't die. Eur J Neurosci 20:3397-3403.

Eisenberg M, Kobilo T, Berman DE, Dudai Y (2003) Stability of retrieved memory: inverse correlation with trace dominance. Science 301:1102-1104.

Everitt BJ, Robbins TW (2000) Second-order schedules of drug reinforcement in rats and monkeys: measurement of reinforcing efficacy and drugseeking behaviour. Psychopharmacology (Berl) 153:17-30.

Everitt BJ, Cardinal RN, Hall J, Parkinson JA, Robbins TW (2000) Differential involvement of amygdala subsystems in appetitive conditioning and drug addiction. In: The amygdala: a functional analysis (Aggleton JP, ed), pp 353-390. Oxford: Oxford UP.

Fuchs RA, Tran-Nguyen LTL, Specio SE, Groff RS, Neisewander JL (1998) Predictive validity of the extinction/reinstatement model of drug craving. Psychopharmacology 135:151-160.

Garavan H, Pankiewicz J, Bloom A, Cho JK, Sperry L, Ross TJ, Salmeron BJ, Risinger R, Kelley D, Stein EA (2000) Cue-induced cocaine craving: neuroanatomical specificity for drug users and drug stimuli. Am J Psychiatry 157:1789-1798.

Gawin FH, Kleber HD (1986) Abstinence symptomatology and psychiatric diagnosis in cocaine abusers. Clinical observations. Arch Gen Psychiatry 43:107-113.

Grant S, London ED, Newlin DB, Villemagne VL, Liu X, Contoreggi C, Phillips RL, Kimes AS, Margolin A (1996) Activation of memory circuits during cue-elicited cocaine craving. Proc Natl Acad Sci USA 93:12040-12045.

Grimm JW, Hope BT, Wise RA, Shaham Y (2001) Neuroadaptation. Incubation of cocaine craving after withdrawal. Nature 412:141-142.

Hatfield T, Han JS, Conley M, Gallagher M, Holland P (1996) Neurotoxic lesions of basolateral, but not central, amygdala interfere with pavlovian second-order conditioning and reinforcer devaluation effects. J Neurosci 16:5256-5265.

Lee JLC, Everitt BJ, Thomas KL (2004) Independent cellular processes for hippocampal memory consolidation and reconsolidation. Science 304:839-843.

Lee JLC, Di Ciano P, Thomas KL, Everitt BJ (2005) Disrupting reconsolidation of drug memories reduces cocaine seeking behavior. Neuron 47:795-801.

Lu L, Hope BT, Dempsey J, Liu SY, Bossert JM, Shaham Y (2005) Central amygdala ERK signaling pathway is critical to incubation of cocaine craving. Nat Neurosci 8:212-219.

Mackintosh N (1974) The psychology of animal learning. London: Academic.

Meil WM, See RE (1996) Conditioned cued recovery of responding following prolonged withdrawal from self-administered cocaine in rats: an animal model of relapse. Behav Pharmacol 7:754-763.

Meil WM, See RE (1997) Lesions of the basolateral amygdala abolish the ability of drug associated cues to reinstate responding during withdrawal from self-administered cocaine. Behav Brain Res 87:139-148.

Milekic MH, Alberini CM (2002) Temporally graded requirement for protein synthesis following memory reactivation. Neuron 36:521-525.

Miller CA, Marshall JF (2005) Molecular substrates for retrieval and reconsolidation of cocaine-associated contextual memory. Neuron 47:873-884.

Nader K, Schafe GE, Le Doux JE (2000) Fear memories require protein synthesis in the amygdala for reconsolidation after retrieval. Nature 406:722-726.

O'Brien CP, Childress AR, Ehrman R, Robbins SJ (1998) Conditioning factors in drug abuse: can they explain compulsion? J Psychopharmacol 12:15-22.

Parkinson JA, Roberts AC, Everitt BJ, Di Ciano P (2005) Acquisition of instrumental conditioned reinforcement is resistant to the devaluation of the unconditioned stimulus. Q J Exp Psychol B 58:19-30.

Robledo P, Robbins TW, Everitt BJ (1996) Effects of excitotoxic lesions of the central amygdaloid nucleus on the potentiation of reward-related stimuli by intra-accumbens amphetamine. Behav Neurosci 110:981-990.

Suzuki A, Josselyn SA, Frankland PW, Masushige S, Silva AJ, Kida S (2004) Memory reconsolidation and extinction have distinct temporal and biochemical signatures. J Neurosci 24:4787-4795.

Taylor JR, Robbins TW (1984) Enhanced behavioural control by conditioned reinforcers following microinjections of $d$-amphetamine into the nucleus accumbens. Psychopharmacology (Berl) 84:405-412.

Thomas KL, Arroyo M, Everitt BJ (2003) Induction of the learning and plasticity-associated gene Zif268 following exposure to a discrete cocaineassociated stimulus. Eur J Neurosci 17:1964-1972.

Valjent E, Corbille AG, Bertran-Gonzalez J, Herve D, Girault JA (2006) Inhibition of ERK pathway or protein synthesis during reexposure to drugs of abuse erases previously learned place preference. Proc Natl Acad Sci USA 103:2932-2937.

Whitelaw RB, Markou A, Robbins TW, Everitt BJ (1996) Excitotoxic lesions of the basolateral amygdala impair the acquisition of cocaine-seeking behaviour underasecond-order schedule of reinforcement. Psychopharmacology 127:213-224. 